Der Reaktionsablauf sei am Bis(phenylethinyl)phosphan ( 1 b) näher diskutiert, da in diesem Fall noch das Produkt (3) isoliert werden konnte, das zusätzliche Hinweise liefert.

Durch intramolekularen nucleophilen Angriff des Phosphors an ein sterisch günstiges Acetylen-C-Atom ${ }^{[3]}$ von (1b) entsteht die Zwischenstufe $\left(1 b^{\prime}\right)$, die sich in Anwesenheit von $\mathrm{H}_{2} \mathrm{O}$ zum Hydroxyphosphoran $\left(1 b^{\prime \prime}\right)$ stabilisieren kann.

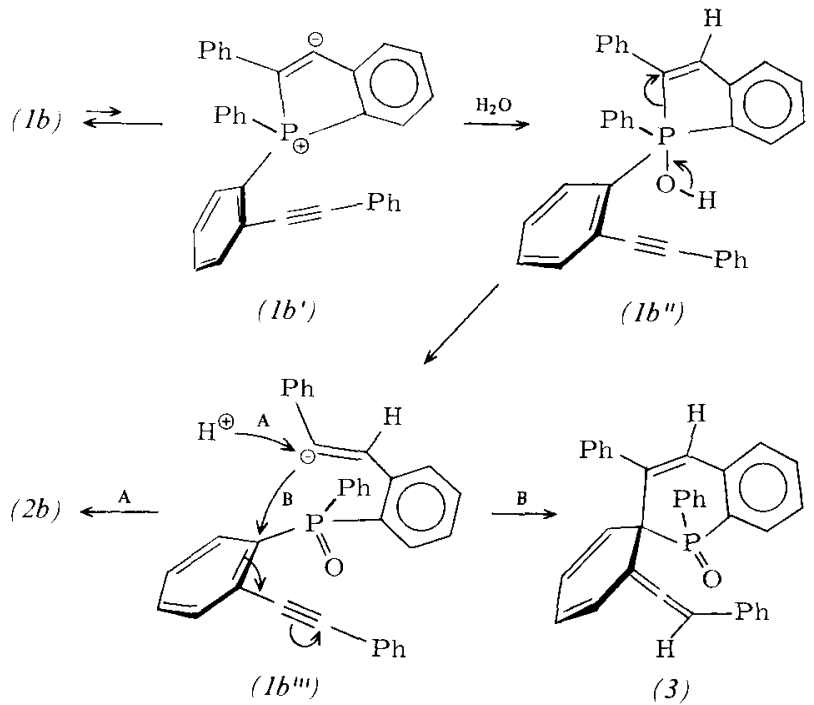

$\left(1 b^{\prime \prime}\right)$ zerfällt unter Spaltung der apicalen $\mathrm{P}-\mathrm{C}_{\text {olefin }}$-Bindung zur Zwischenstufe $\left(1 b^{\prime \prime \prime}\right)$, die durch Protonierung des carbanionischen Olefin-C-Atoms das stabile Endprodukt ( $2 b$ ) liefert (Weg A). Das carbanionische Olefin-C-Atom kann aber auch einen Benzolring nucleophil angreifen und zu Verbindung (3) mit einem Spiro-Cyclohexadien-Allen-Strukturelement führen (Weg B).

In Anwesenheit von $\mathrm{HCl}$ entsteht nur ( $2 b$ ); offensichtlich verläuft die Protonierung des Carbanion-C-Atoms schneller als dessen nucleophile Addition an den Benzolring. Die ungewöhnliche Struktur von (3) wurde durch Röntgen-Strukturanalyse $\mathrm{e}^{[4]}$ gesichert.

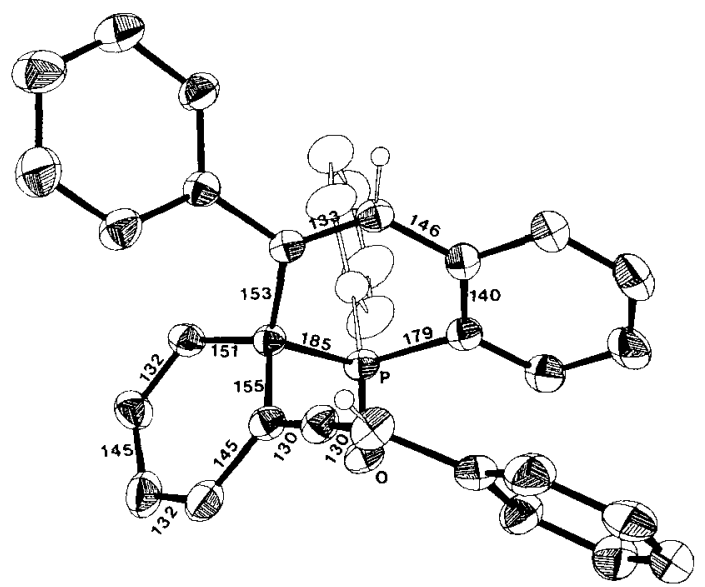

Abb. 1. Molekülstruktur von (3). Der Übersichtlichkeit halber wurden nur die ,addierten" H-Atome gezeichnet. Die Standardabweichungen der Bindungslängen (in pm) betragen im Mittel $0.5 \mathrm{pm}[4]$.

Die Reaktion $(1) \rightarrow(2)$ erscheint in zweifacher Hinsicht bemerkenswert: Durch einfaches „Umkristallisieren“ können die $o$-Phenylethinyl-substituierten Triphenylphosphane ( $1 a$ )(1c) stereospezifisch in Triphenylphosphanoxide mit transStilben- und Tolan-Strukturelementen umgewandelt werden. Durch Reduktion dieser Phosphanoxide (2) mit $\mathrm{SiHCl}_{3}{ }^{[5]}$ entstehen neue Übergangsmetall-Liganden mit Phosphan-, Acetylen- und Olefin-Donorstellen innerhalb eines Moleküls. Weiterhin repräsentiert der Reaktionsweg B eines der wenigen Beispiele ${ }^{[6]}$, in denen nach apicaler Ringöffnung eines cycli- schen Hydroxyphosphorans ein Phenylsubstituent von der Abgangsgruppe nucleophil angegriffen wird.

Der ausschließliche Angriff des Phenylethinyl-substituierten Phenylrings in $\left(1 b^{\prime \prime}\right)$ dïrfte dadurch zu erklären sein, daß sich ein konjugationsfähiges Cyclohexadien-Allen-System bilden kann.

Eingegangen am 19. September 1978 [Z 103]

[1] a) W. Winter, Angew. Chem. 88, 260 (1976); Angew. Chem. Int. Ed. Engl. 15, 241 (1976); b) Synthese von (1b): W. Winter, Chem. Ber. 109, 2405 (1976); Synthese von $(1 c)$ : analog $(1 b)$.

[2] ( $2 a)$ : Ausb. $76 \%$, farblose Kristalle, $\mathrm{Fp}=169-170^{\circ} \mathrm{C}$ (Cyclohexan/Benzol); IR: $1195(\mathrm{P}=\mathrm{O})$ und $960 \mathrm{~cm}^{-1}(\mathrm{C}-\mathrm{H}$ out-of-plane für trans-Olefin); ${ }^{1} \mathrm{H}-\mathrm{NMR}$ (TMS, $\mathrm{CDCl}_{3}$ ): Olefin-AB-System: $\delta_{\mathrm{A}}=7.83, \quad \delta_{\mathrm{B}}=6.87$, $J_{\mathrm{AB}}=16 \mathrm{~Hz} ;(2 \mathrm{~b})$ : Ausb. $54 \%$, farblose Kristalle, $\mathrm{Fp}=160-161^{\circ} \mathrm{C}$ (EtOH); IR: $2225(-\mathrm{C} \equiv \mathrm{C}-), 1190(\mathrm{P}=\mathrm{O})$ und $965 \mathrm{~cm}^{-1}$ (trans-Olefin); ${ }^{1} \mathrm{H}-\mathrm{NMR}$ (TMS, $\mathrm{CDCl}_{3}$ ): Olefin-AB-System: $\dot{\delta}_{\mathrm{A}}=7.80, \quad \delta_{\mathrm{B}}=6.83$, $J_{A B}=16 \mathrm{~Hz}$; (2c): Ausb. $88 \%$, farblose Kristalle, $\mathrm{Fp}=205-207^{\circ} \mathrm{C}$ (EtOH); IR: $2210(-\mathrm{C} \equiv \mathrm{C}-), 1190(\mathrm{P}-\mathrm{O})$ und $950 \mathrm{~cm}^{-1}$ (trans-Olefin); ${ }^{1} \mathrm{H}-\mathrm{NMR}$ (TMS, $\mathrm{CDCl}_{3}$ ): Olefin-AB-System: $\delta_{\mathrm{A}}=7.62, \quad \delta_{\mathrm{B}}=6.38$, $J_{\mathrm{AB}}=16 \mathrm{~Hz}$.

[3] D. W. Allen, J. C. Tebby, Tetrahedron 23, 2795 (1967).

[4] Monoklin, Raumgruppe P2 $1 \mathrm{c} ; \quad a=873.7(2), \quad b=1817.4(10)$ $c=1639.8(5) \mathrm{pm}, \beta=104.16(2)^{\circ} ; \mathrm{Z}=4 ; 2413$ symmetrieunabhängige Reflexe $[\mathrm{I} \geq 2 \sigma(\mathrm{I})]$, Nonius CAD-4, Mo $\mathrm{K}_{\alpha}$-Graphitmonochromator; Bestimmung der Phasen mit direkten Methoden (SHELX); Verfeinerung: $\mathrm{R}=0.042$ ( $\mathrm{P}, \mathrm{O}, \mathrm{C}$ anisotrop, $\mathrm{H}$ isotrop).

[5] W. Winter, noch unveröffentlicht.

[6] S. E. Fishwick, J. Flint, W. Hawes, S. Tripett, Chem. Commun. 1967 1113 ; S. E. Cremer, ibid. 1968, 1132; D. Hellwinkel, H.-J. Wilfinger, Chem. Ber. 105, 3878 (1972).

\section{Redoxeigenschaften von Zink-tetrabenzporphyrin $\left.{ }^{[*}{ }^{*}\right]$}

Von Arnd Vogler, Birgit Rethwisch, Horst Kunkely, Jürgen Hüttermann und Jürgen Otto Besenhard ${ }^{[*]}$

Elektronenübertragungen unter Beteiligung von Metalloporphyrinen spielen eine wichtige Rolle bei der Photosynthese und anderen biologischen Redoxreaktionen. Die Redoxpotentiale von Porphyrinen können bisher im wesentlichen nur durch Variation des Zentralmetalls und zusätzlicher axialer Liganden verändert werden ${ }^{[1]}$. Als dritte Möglichkeit bietet sich die Modifizierung des Porphyrinringes an. Während einfache Substituenten die Redoxeigenschaften nur geringfügig beeinflussen, könnte eine Erweiterung des $\pi$-Elektronensystems von Porphyrinen zu einer beträchtlichen Senkung der Redoxpotentiale führen ${ }^{[1]}$. Am Beispiel von Tetrabenzporphyrin (TBP) haben wir gefunden, daß diese Erwartung zutrifft. Wir berichten über das Redoxverhalten von Zink-tetrabenzporphyrin, ZnTBP $(1)^{[21}$, das ein redoxinertes Metallzentrum enthält.

Die Ergebnisse der zyklischen Voltammetrie sollen anhand des Reaktionsschemas

$$
\begin{gathered}
{\left[\mathrm{Zn}\left(\mathrm{TBP}^{-}\right)\right]^{\ominus} \underset{+\mathrm{e}}{\stackrel{-\mathrm{e}}{\rightleftarrows}}[\mathrm{Zn}(\mathrm{TBP})] \stackrel{-\mathrm{e}}{\rightleftarrows}} \\
(4) \\
{\left[\mathrm{Zn}\left(\mathrm{TBP}^{+}\right)\right]^{\oplus} \underset{+\mathrm{c}}{\stackrel{-\mathrm{e}}{\rightleftarrows}}\left[\mathrm{Zn}\left(\mathrm{TBP}^{2+}\right)\right]^{2^{\oplus}}}
\end{gathered}
$$

erläutert werden. (1) läßt sich in zwei reversiblen Einelektronenschritten zu (2) und (3) oxidieren, $E_{1 / 2}=+0.36$ bzw.

[*] Prof. Dr. A. Vogler, Dr. H. Kunkely, B. Rethwisch

Institut für Chemie der Universität

Universitätsstraße 31, D-8400 Regensburg 2

Prol. Dr. J. Hüttermann

Institut für Biophysik und Physikalische Biochemie der Universität Regensburg

Dr. J. O. Besenhard

Anorganisch-chemisches Institut der Technischen Universität München Lichtenbergstraße 4, D-8046 Garching

[**] Diese Arbeit wurde von der Deutschen Forschungsgemeinschaft und vom Fonds der Chemischen Industrie unterstützt. 
$+0.97 \mathrm{~V}$ (rel. SCE, an $\mathrm{Pt}$ in $1 \mathrm{M} \mathrm{LiClO}_{4} / \mathrm{DMSO}$ ). Die erste Reduktion von (1) zu (4) bei $E_{1 / 2}=-1.48 \mathrm{~V}$ ist ebenfalls reversibel. Mit $1.84 \mathrm{~V}$ weist TBP also eine wesentlich geringere Potentialdifferenz zwischen erster Ringoxidation und -reduktion auf als andere Porphyrine, bei denen diese Differenz in der Regel ca. $2.25 \mathrm{~V}$ beträgt ${ }^{[1]}$. Bemerkenswert ist das niedrige erste Oxidationspotential von (1) $\left(E_{1 / 2}=+0.36 \mathrm{~V}\right)$; Zinkoctaethylporphyrin und -tetraphenylporphyrin werden erst bei $E_{1 / 2}=+0.63^{[3]}$ bzw. $+0.71 \mathrm{~V}^{[4]}$ oxidiert.

Da Porphyrin- $\pi$-Radikalionen besonderes Interesse zukommt ${ }^{[5]}$, wurde die Spezies (2) näher untersucht ${ }^{[6]}$. Die Oxidation von (1) mit Iod in Methanol/Dimethylsulfoxid (10:1) ergibt (2), dessen Absorptionsspektrum (Abb. 1) typisch für Porphyrin- $\pi$-Radikalkationen ${ }^{[1,5]}$ ist. (2) kann (z.B. mit Dithionit) wieder vollständig zu (1) reduziert werden.

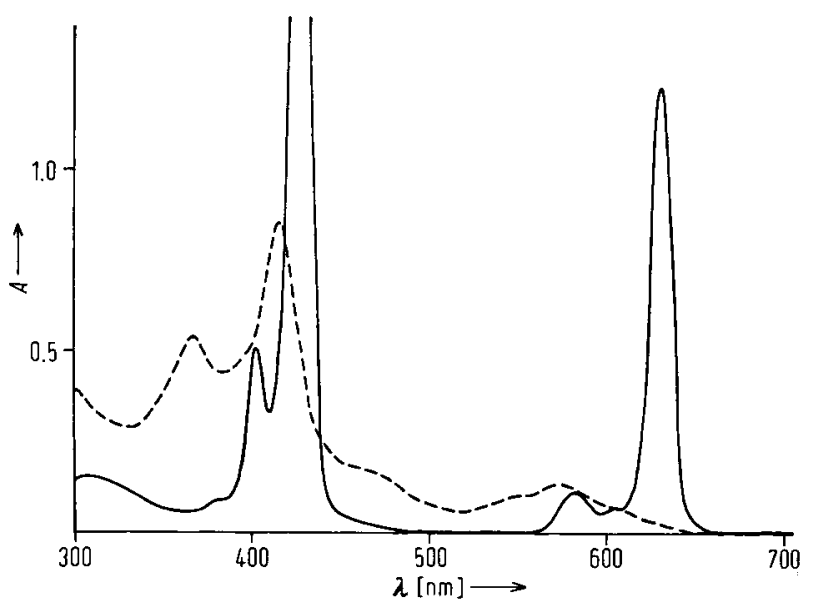

Abb. 1. Absorptionsspektrum von ZnTBP (--), $8.13 \cdot 10^{-5} \mathrm{~mol} / \mathrm{l}$ in Methanol/Dimethylsulfoxid (10:1), $1 \mathrm{~mm}$-Küvette; nach der Oxidation mit Iod (----).

Zur weiteren Charakterisierung von (2) diente die ESRSpektroskopie: In Methanol/Pyridin (10:1) zeigt (2) schon bei Raumtemperatur eine einzelne Linie mit einer Breite von $5.5 \mathrm{G}$ und einem g-Wert von 2.0029. Auch bei $77 \mathrm{~K}$ konnte keine Hyperfeinstruktur beobachtet werden.

Eingegangen am 25. September $1978 \quad$ [Z 105a]

[1] J.-H. Fuhrhop in K. M. Smith: Porphyrins and Metalloporphyrins. Elsevier, Amsterdam 1975, Kap. 14.

[2] Synthese von (1): A. Vogler, H. Kunkely, Angew. Chem. 90, 808 (1978); Angew. Chem. Int. Ed. Engl. 17, 760 (1978).

[3] J.-H. Fuhrhop, K. Kadish, D. G. Davis, J. Am. Chem. Soc. 95, 5140 (1973).

[4] A. Stanienda, Z. Phys. Chem. (Leipzig) 229, 259 (1964)

[5] D. Dolphin, R. H. Felton, Acc. Chem. Res. 7, 26 (1974).

[6] Die Oxidation von MgTBP wurde erstmals beobachtet von J. C. Goedheer, Photochem. Photobiol. 6, 521 (1967).

\section{Carbonyl(pyridin)eisen(II)-tetrabenzporphyrin- $\pi$-Radikalkation $\left.{ }^{[* *}\right]$}

Von Arnd Vogler, Birgit Rethwisch, Horst Kunkely und Jürgen Hüttermann ${ }^{[*]}$

Eisenporphyrine fungieren als Elektronenüberträger in biologischen Systemen. Bei Cytochromen verläuft der Elektro-

[*] Prof. Dr. A. Vogler, Dr. H. Kunkely, B. Rethwisch

Institut für Chemie der Universität

Universitätsstraße 31, D-8400 Regensburg 2

Prof. Dr. J. Hüttermann

Institut für Biophysik und Physikalische Biochemie der Universität Regensburg

[**] Diese Arbeit wurde von der Deutschen Forschungsgemeinschaft und vom Fonds der Chemischen Industrie unterstützt. nentransfer über das Redoxpaar $\mathrm{Fe}^{\mathrm{II}} / \mathrm{Fe}^{\mathrm{III}}$. Es gibt aber Hinweise darauf, daß im ersten Schritt solcher Redoxreaktionen ein $\pi$-Elektron des Porphyrinrings beteiligt ist ${ }^{[1]}$. Während die Oxidation einiger $\mathrm{Ru}^{\mathrm{II}}$-Porphyrine tatsächlich zum Verlust eines Porphyrinelektrons unter Bildung stabiler Porphyrin- $\pi$ Radikalkationen führt ${ }^{[2]}$, wurden nach der Einelektronenoxidation von $\mathrm{Fe}^{\mathrm{II}}$-Porphyrinen bisher nur die entsprechenden $\mathrm{Fe}^{\mathrm{III}}$-Komplexe beobachtet ${ }^{[3]}$. Die Erklärung dafür ist, daß $\mathrm{Fe}^{\mathrm{II}}$ bei niedrigeren und $\mathrm{Ru}^{\mathrm{II}}$ bei höheren Potentialen als der Porphyrinring oxidiert wird. Wenn es gelingt; das Oxidationspotential eines Porphyrins unter das von $\mathrm{Fe}^{\mathrm{II}}$ zu senken, sollte es möglich sein, ein stabiles Fe ${ }^{\text {II }}$-Porphyrin- $\pi$-Radikalkation zu erhalten. Die Existenz einer solchen Verbindung wäre nicht nur interessant im Zusammenhang mit dem Mechanismus biologischer Redoxreaktionen. Ein $\mathrm{Fe}^{\mathrm{II}}$-Porphyrin- $\pi-\mathrm{Ra}$ dikalkation ist isomer (,Oxidationsstufenisomerie" ${ }^{\text {[3] }}$ ) zum entsprechenden $\mathrm{Fe}^{\mathrm{III}}$-Porphyrin; beide Formen unterscheiden sich nur durch die Lokalisierung eines Elektrons. Im Falle der bisher bekannten „low spin"-Fe ${ }^{\mathrm{III}}$-Porphyrine ist das isomere $\mathrm{Fe}^{\mathrm{II}}$-Porphyrin- $\pi$-Radikalkation ein bei niedrigen Energien liegender elektronisch angeregter CT-Zustand ${ }^{[4]}$, der im Absorptionsspektrum zu beobachten ist ${ }^{[5]}$. In einem stabilen $\mathrm{Fe}^{\mathrm{II}}$-Porphyrin- $\pi$-Radikalkation wird dieser CT-Zustand zum neuen Grundzustand.

Wir haben jetzt gefunden, daß die Oxidation von $\left[\mathrm{Fe}^{\mathrm{II}}(\mathrm{TBP})\right.$ (CO)(py)] (1) $(\mathrm{TBP}=$ Tetrabenzporphyrin) tatsächlich zum Verlust eines $\pi$-Elektrons aus dem Porphyrinliganden unter Bildung von $\left[\mathrm{Fe}^{\mathrm{II}}\left(\mathrm{TBP}^{+}\right)(\mathrm{CO})(\mathrm{py})\right]^{\oplus}$ (2) fuihrt. Folgende Kriterien bestimmten die Auswahl von (1): TBP besitzt ein sehr niedriges Oxidationspotential ${ }^{[6]}$, und der CO-Ligand stabilisiert $\mathrm{Fe}^{\mathrm{II}}$ gegenüber $\mathrm{Fe}^{\mathrm{III}[3]}$; daher wird die Oxidation von $\mathrm{Fe}^{\mathrm{II}} \mathrm{zu}$ höheren Potentialen verschoben.

(1) wurde durch Lösen von $\left[\mathrm{Fe}^{\mathrm{II}}(\mathrm{TBP})(\mathrm{py})_{2}\right]^{[7]}$ in $\mathrm{CHCl}_{3}$, gesättigt mit $\mathrm{CO}$, erhalten. Das IR-Spektrum von (1) in $\mathrm{CHCl}_{3}$ zeigt bei $1975 \mathrm{~cm}^{-1}$ eine CO-Valenzschwingung. Oxidation mit Iod ergibt (2), dessen Absorptionsspektrum (Abb. 1) für die Porphyrinoxidation charakteristisch ist. Im IR-Spektrum von (2) erscheint bei $2020 \mathrm{~cm}^{-1}$ eine vCO-Bande. Fe ${ }^{\mathrm{III}}$-Carbonylkomplexe sind nicht bekannt. Die Bandenverschiebung um $45 \mathrm{~cm}^{-1}$ durch die Oxidation von (1) sollte von der positiven Gesamtladung von (2) herrühren.

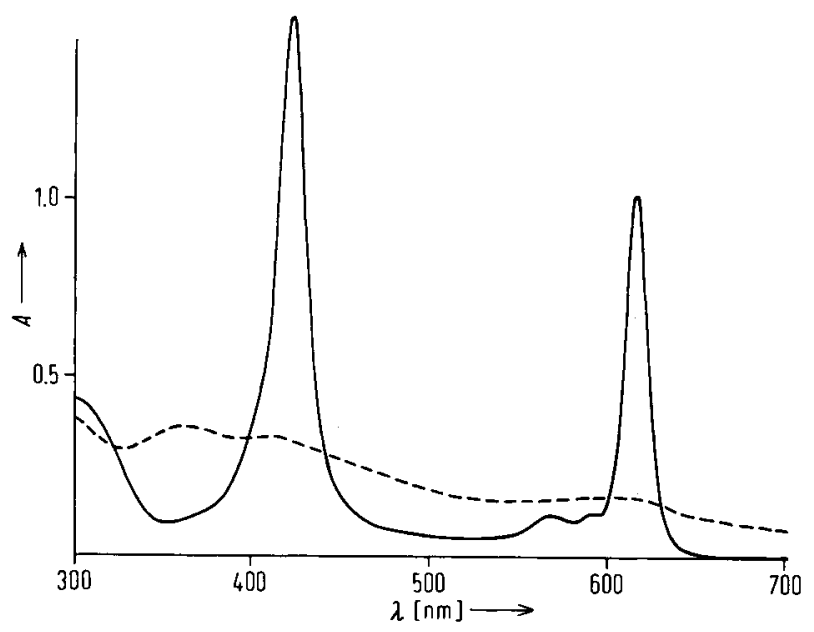

Abb. 1. Absorptionsspektrum von $\operatorname{FeTBP}(\mathrm{CO})(\mathrm{py})(-), 1.1 \cdot 10^{-5} \mathrm{~mol} / \mathrm{l}$ in $\mathrm{CHCl}_{3}, 1 \mathrm{~cm}-\mathrm{Küvette}$; nach der Oxidation mit Iod (------).

Das ESR-Spektrum von (2) in $\mathrm{CHCl}_{3} / \mathrm{Methanol}(1: 1)$ zeigt eine einzelne Linie mit einer Breite von $5.0 \mathrm{G}$ und einem g-Wert von 2.0032. Dieses Signal, das allerdings nur unterhalb $77 \mathrm{~K}$ deutlich auftritt, ist dem von $\left[\mathrm{Zn}\left(\mathrm{TBP}^{+}\right)\right]^{\oplus} \mathrm{sehr}$ ähnlich ${ }^{[6]}$ und unterscheidet sich völlig von den ESR-Spektren der Fe ${ }^{\mathrm{III}}$. 